\title{
Infiltración de resina y barniz de flúor para el tratamiento de caries interproximales no cavitadas en dentición permanente.
}

\section{Resin infiltration plus fluoride varnish for non-cavitated proximal caries in permanent dentition.}

\begin{abstract}
Ana María Rojas-Gómez ${ }^{1,2^{*}}$, Francisca Verdugo-Paiva ${ }^{3,4}$, Julián Balanta-Melo ${ }^{5,6}$
1. Departamento de Epidemiología Clínica y Bioestadística. Facultad de Medicina, Pontificia Universidad Javeriana, Bogotá, Colombia 2. Unidad de Investigación en Medicina Estomatológica Preventiva y Social (UIMEPS). Programa de odontología, Universidad del Magdalena, Santa Marta, Colombia.

3. Centro Evidencia UC, Facultad de Medicina, Pontificia Universidad Católica de Chile, Santiago, Chile

4. Proyecto Epistemonikos, Santiago, Chile

5. Escuela de Odontología, Facultad de salud,

Universidad del Valle, Cali, Colombia

6. Centro asociado Cochrane, Facultad de

Odontología, Universidad de Chile, Santiago, Chile

* Correspondencia Autor: Ana María Rojas Gómez | Dirección: Centro Evidencia UC, Pontificia Universidad Católica de Chile, Diagonal Paraguay 476, Santiago, Chile | E-mail: anrojget@gmail.com

RESUMEN

Introducción: Para evitar la progresión de la caries interproximal no cavitada en dientes permanentes, convencionalmente se utilizan técnicas mínimamente invasivas. Dentro de las técnicas más utilizadas destacan la aplicación tópica de barniz de flúor, sellantes o la infiltración de resina. Sin embargo, aún existe incertidumbre respecto a la efectividad de la infiltración en resina y el barniz de flúor cuando se usan en conjunto en dentición permanente. Métodos: Realizamos una búsqueda en Epistemonikos, la mayor base de datos de revisiones sistemáticas en salud, la cual es mantenida mediante el cribado de múltiples fuentes de información, incluyendo MEDLINE, EMBASE, Cochrane, entre otras. Extrajimos los datos desde las revisiones identificadas, analizamos los datos de los estudios primarios, realizamos un metaanálisis y preparamos una tabla de resumen de los resultados utilizando el método GRADE. Resultados y conclusiones: Se identificaron 11 revisiones sistemáticas que en conjunto incluyeron cuatro estudios primarios que corresponden a ensayos clínicos aleatorizados. Se concluye que la infiltración de resina probablemente reduce la progresión de la caries interproximal no cavitada en dientes permanentes. No se encontraron estudios que evaluaran los eventos adversos.
\end{abstract}

\section{PALABRAS CLAVE}

Caries; Infiltración de resina; Caries interproximal; Barniz de flúor; Tratamiento mínimamente invasivo; Epistemonikos; GRADE.

\begin{abstract}
Introduction: Minimally invasive techniques are conventionally used to prevent the progression of non-cavitated interproximal caries in permanent teeth. Topical fluoride varnish, sealants, and resin infiltration are the most common techniques. However, there is still uncertainty about the effectiveness of resin infiltration in conjunction with fluoride varnish in permanent dentition. Methods: We searched in Epistemonikos, the largest database of systematic reviews in health, which is maintained by screening multiple information sources, including MEDLINE, EMBASE, Cochrane, among others. We extracted data from the systematic reviews, reanalyzed data of primary studies, conducted a meta-analysis and generated a summary of findings table using the GRADE approach. Results and conclusions: We identified 11 systematic reviews including four studies overall, of which all were randomized trials. We conclude that resin infiltration probably decreases the risk of progression of the risk of non-cavitated interproximal caries in permanent dentition. No studies were found that looked at adverse effects.
\end{abstract}

\section{KEY WORDS}

Caries treatment; Resin infiltration; Proximal lesion; Fluoride varnish; Micro-invasive treatment; Permanent teeth; Epistemonikos; GRADE.

Int. J. Inter. Dent Vol. 14(1); 105-109, 2021. 


\section{PROBLEMA}

La caries dental se manifiesta principalmente ante la presencia de una desmineralización en los tejidos dentarios prolongada que conduce a la cavitación de las lesiones cariosas ${ }^{(1)}$. Cuando la lesión cariosa inicia sin cavitar el esmalte, puede ser tratada con técnicas mínimamente invasivas como la aplicación tópica de barniz de flúor y la infiltración de resina, las cuales evitan la intervención mecánica del tejido dental afectado conservando la estructura del diente y la salud de la pulpa ${ }^{(2,3)}$.

La infiltración de resina en lesiones cariosas no cavitadas utiliza fuerzas capilares para transportar resinas metacrílicas con altos coeficientes de penetración en el esmalte poroso interproximal, deteniendo el proceso de desmineralización evitando la progresión a una lesión cavitada ${ }^{(4,5)}$

Se sugiere que la aplicación tópica de barniz de flúor es más efectiva cuando se combina con la técnica de infiltración de resina, evitando la progresión de la caries interproximal no cavitada ${ }^{(6,7)}$, sin embargo existe aún incertidumbre respecto de su efectividad en dientes permanentes.

\section{MÉTODOS}

Realizamos una búsqueda en Epistemonikos, la mayor base de datos de revisiones sistemáticas en salud, la cual es mantenida mediante búsquedas en múltiples fuentes de información, incluyendo MEDLINE, EMBASE, Cochrane, entre otras. Extrajimos los datos desde las revisiones identificadas y analizamos los datos de los estudios primarios. Con esta información, generamos un resumen estructurado denominado FRISBEE (Friendly Summaries of Body of Evidence using Epistemonikos), siguiendo un formato preestablecido, que incluye mensajes clave, un resumen del conjunto de evidencia (presentado como matriz de evidencia en Epistemonikos), metanálisis del total de los estudios cuando sea posible, una tabla de resumen de resultados con el método GRADE y una sección de otras consideraciones para la toma de decisión.

\section{Mensajes clave}

- El uso de la infiltración de resina más el barniz de flúor probablemente reduce el riesgo de la progresión de caries en lesiones interproximales no cavitadas en dientes permanentes.

- No se encontraron estudios que evaluaran los eventos adversos.

(20)

Todos los ensayos tuvieron un diseño de boca dividida y evaluaron la aplicación de la infiltración de resina más barniz de flúor en comparación a la aplicación única de flúor barniz ${ }^{(17-21)}$

En el grupo que recibió infiltración de resina fue aplicada usando aislamiento absoluto y una cuña en los estudios incluidos. En todos los estudios indican que realizaron el grabado con ácido hidroclorhídrico al $15 \%$ por 120 segundos; seguido de lavado, secado, deshidratación con etanol y nuevamente secado por 10 segundos $^{(17-19)}$ O 30 segundos $^{(20)}$. Posteriormente inician con la

Qué tipo de intervenciones incluyeron los estudios* infiltración de resina de fotocurado, polimerizándola dos veces. El producto usado en los estudios es ICON de la marca DMG (Hamburgo, Alemania). En relación con la aplicación de barniz de flúor indican que este fue posterior a la infiltración de resina sin dar detalles del protocolo.

Por su parte, todos los ensayos utilizaron placebo (Agua en jeringa con aislamiento absoluto). Ninguno de los estudios indica la marca del barniz de flúor. Todos los ensayos realizaron además educación oral e instrucciones en la dieta a todos sus participantes ${ }^{(17-21)}$

Los ensayos reportaron múltiples desenlaces, los cuales fueron agrupados por las revisiones sistemáticas de la siguiente manera:

Qué tipo de desenlaces midieron

- Progresión de caries dental

El seguimiento promedio de los ensayos fue de entre 18 y 36 meses $^{(17-21)}$.

* La información sobre los estudios primarios es extraída desde las revisiones sistemáticas identificadas, no directamente desde los estudios, a menos que se especifique lo contrario.

\section{RESUMEN DE LOS RESULTADOS}

La información sobre los efectos de la infiltración de resina más el barniz de flúor en dientes permanentes con caries interproximales se basa en cuatro ensayos clínicos aleatorizados que incluyeron 596 lesiones en 173 pacientes.

Dos ensayos evaluaron el desenlace progresión de caries hasta 18 meses (426 dientes) $)^{(17,19)}$ y 3 ensayos evaluaron el desenlace progresión de caries entre 18 y 36 meses (170 dientes) ${ }^{(17,20,21)}$.

El resumen de los resultados es el siguiente:

- El uso de la infiltración de resina más el barniz de flúor probablemente reduce el riesgo de la progresión de caries en lesiones interproximales no cavitadas en dientes permanentes en periodos de seguimiento $18 \leq$ meses

- El uso de la infiltración de resina más el barniz de flúor probablemente reduce el riesgo de la progresión de caries en lesiones interproximales no cavitadas en dentición permanente en períodos de seguimiento entre 18 -36 meses

- No se encontraron estudios que evaluaran los eventos adversos 


\section{OTRAS CONSIDERACIONES PARA LA TOMA DE DECISIÓN}

\section{A quién se aplica y a quién no se aplica esta evidencia}

- Los resultados de este resumen se aplican a pacientes con lesiones interproximales no cavitadas en dientes posteriores permanentes.

- Los resultados de esta evidencia no aplican a pacientes embarazadas debido al posible riesgo de fetotoxicidad, anomalías del desarrollo y posible resistencia al cloruro de hidrógeno por inhalación durante el embarazo ${ }^{(22)}$

\section{Sobre los desenlaces incluidos en este resumen}

- El desenlace de progresión de caries es considerado crítico para la toma de decisión, de acuerdo con la opinión de los autores de este resumen. Además, coinciden con los evaluados por las revisiones sistemáticas identificadas.

- Los autores consideran que el desenlace "eventos adversos" es relevante para la toma de decisiones, sin embargo, no fueron medidos por las revisiones sistemáticas.

\section{Balance daño/beneficio y certeza de la evidencia}

- La evidencia incluida en este resumen muestra un beneficio en la reducción del riesgo de progresión de caries usando resina infiltrativa más barniz de flúor comparado con usar solo barniz de flúor en dentición permanente a los $18 \leq$ y de $18>-36$ meses

- Las revisiones sistemáticas incluidas en este resumen no reportan eventos adversos en el uso de la resina infiltrativa, no obstante, para el uso de esta técnica es obligatorio el uso aislamiento absoluto para reducir el riesgo de complicaciones por el uso del del ácido hidroclorhídrico. Así mismo, algunos autores, considerando los efectos ambientales, recomiendan siempre inactivar el ácido hidroclorhídrico con bicarbonato de sodio inmediatamente después del tiempo de aplicación en la lesión y antes de realizar la succión ${ }^{(23)}$

- A pesar de que se considera que la infiltración de resina presentaría bajo riesgo de complicaciones usando aislamiento absoluto, existe incertidumbre sobre el efecto del ácido hidroclorhídrico $(\mathrm{HCl})$ sobre la estructura dental y pulpar ${ }^{(16)}$

- En base a lo anterior, el balance entre beneficios y riesgos debería evaluarse de manera individualizada con los pacientes y sus cuidadores.

\section{Consideraciones de recursos}

- Ninguna de las revisiones sistemáticas consideró dentro de sus desenlaces un análisis económico.

- La infiltración de resina más barniz de flúor podría reducir el costo total del tratamiento ya que esta intervención combinada se aplica en una única sesión, mientras que si se utiliza flúor barniz por sí solo requiere ser aplicado en varias ocasiones. Además al reducir el riesgo de progresión de caries, reduce el costo para el tratamiento de la lesión que avanza a cavitación ${ }^{(24,25)}$

- Los costos del barniz de flúor son menores a los generados por el kit para la infiltración de resina, lo que podría influenciar al clínico en la toma de decisión.

\section{Qué piensan los pacientes y sus tratantes}

- En general, el uso de tratamientos mínimamente invasivos como la infiltración de resina más el barniz de flúor son bien recibidas tanto por los pacientes como por los tratantes, teniendo mayor aceptabilidad en comparación a los tratamientos invasivos donde se remueven de manera mecánica el tejido con caries debido al enfoque conservador.

- Los pacientes tienden a expresar experiencias negativas relacionadas al dolor, la aplicación de anestésico, el aislamiento absoluto y demás elementos en el tratamiento convencional de caries ${ }^{(26)}$. Estos últimos elementos también están presentes en la infiltración de resina, lo que podría también generar experiencias negativas para cierto tipo de pacientes o podría condicionar la toma de decisiones clínicas del operador

\section{Diferencias entre este resumen y otras fuentes}

- Las conclusiones de este análisis coinciden con lo encontrado por cada una de las revisiones sistemáticas identificadas ${ }^{(6-16)}$ las cuales consideran que el uso de la infiltración de resina para el tratamiento de la caries interproximal no cavitada disminuye el riesgo de progresión de caries en dientes posteriores permanentes.

- Así mismo coincide con las recomendaciones dadas desde distintas la guía clínica para el manejo de la caries de la International Caries Classification and Management System ${ }^{(27)}$.

\section{¿Puede que cambie esta información en el futuro?}

- La información presentada en este resumen para el desenlace de progresión de caries podría cambiar si aparece nueva investigación en el futuro, debido a la certeza de la evidencia ya existente.

- Identificamos seis revisiones sistemáticas en curso en el International prospective register of systematic reviews (PROSPERO) (28-33) y un ensayo clínico en la International Clinical Trials Registry Platform de la Organización Mundial de la Salud (34) que podrían aportar información adicional relevante con relación a la eficacia del uso de la infiltración de resina más el barniz de flúor.

\section{CÓMO REALIZAMOS ESTE RESUMEN}

Mediante métodos automatizados y colaborativos recopilamos toda la evidencia relevante para la pregunta de interés y la presentamos en una matriz de evidencia.

Siga el enlace para acceder a la versión interactiva: Infiltración de resina comparado con barniz de flúor para caries no cavitadas proximales en dentición permanente

\section{NOTAS}

Si con posterioridad a la publicación de este resumen se publican nuevas revisiones sistemáticas sobre este tema, en la parte superior de la matriz se mostrará un aviso de "nueva evidencia".

Este artículo es parte del proyecto síntesis de evidencia de Epistemonikos. Se elabora con una metodología preestablecida, siguiendo rigurosos estándares metodológicos y proceso de revisión por pares interno. Cada uno de estos artículos corresponde a un resumen, denominado FRISBEE (Friendly Summary of Body of Evidence using Epistemonikos), cuyo principal objetivo es sintetizar el conjunto de evidencia de una pregunta específica, en un formato amigable a los profesionales clínicos. Sus principales recursos se basan en la matriz de evidencia de Epistemonikos y análisis de resultados usando metodología GRADE. Mayores detalles de los métodos para elaborar este FRISBEE están descritos aquí (http://dx.doi.org/10.5867/medwave.2014.06.5997)

La Fundación Epistemonikos es una organización que busca acercar la información a quienes toman decisiones en salud, mediante el uso de tecnologías. Su principal desarrollo es la base de datos Epistemonikos (www.epistemonikos.org).

\section{DECLARACIÓN DE CONFLICTOS DE INTERESES}

Los autores declaran no tener conflictos de intereses con la materia de este artículo.

\section{AGRADECIMIENTOS}

Este resumen de evidencia fue elaborado con el apoyo metodológico del Centro Evidencia UC, Facultad de Medicina, Pontificia Universidad Católica de Chile. 


\section{Bibliografía}

1. Kidd Edwina AM. Essentials of dental caries: The disease and its management. Oxford USA: Oxford University Press, 2005.

2. Nielsen LA. Caries progression in the deciduous teeth from 3 to 7 years of age. Tandlaegebladet. 2001;(9):704-11.

3. Schwendicke F, Frencken JE, Bjørndal L, Maltz M, Manton DJ, Ricketts D, et al. Managing carious lesions: consensus recommendations on carious tissue removal. Adv Dent Res. 2016;28(2):58-67.

4. Meyer-Lueckel H, Paris S, Kielbassa AM. Surface layer erosion of natural caries lesions with phosphoric and hydrochloric acid gels in preparation for resin infiltration. Caries Res. 2007;41(3):223-30.

5. Paris S, Meyer-Lueckel H, Cölfen H, Kielbassa AM. Resin infiltration of artificial enamel caries lesions with experimental light curing resins. Dent Mater J. 2007 Jul;26(4):582-8.

6. Dorri M, Dunne SM, Walsh T, Schwendicke F. Micro-invasive interventions for managing proximal dental decay in primary and permanent teeth. Cochrane Database Syst Rev. 2015 Nov;(11):CD010431.

7. Doméjean S, Ducamp R, Léger S, Holmgren C. Resin infiltration of non-cavitated caries lesions: a systematic review. Med Princ Pract Int J Kuwait Univ Heal Sci Cent. 2015;24(3):216-21.

8. Splieth $\mathrm{CH}$, Kanzow P, Wiegand A, Schmoeckel J, Jablonski-Momeni A. How to intervene in the caries process: proximal caries in adolescents and adults-a systematic review and meta-analysis. Clin Oral Investig. 2020;24(5):1623-36.

9. Krois J, Göstemeyer G, Reda S, Schwendicke F. Sealing or infiltrating proximal carious lesions. J Dent. 2018 Jul;74:15-22.

10. Ammari MM, Soviero VM, da Silva Fidalgo TK, Lenzi M, Ferreira DMTP, Mattos $\mathrm{CT}$, et al. Is non-cavitated proximal lesion sealing an effective method for caries control in primary and permanent teeth? A systematic review and meta-analysis. J Dent. 2014 Oct:42(10):1217-27.

11. Urquhart O, Tampi MP, Pilcher L, Slayton RL, Araujo MWB, Fontana M, et al Nonrestorative treatments for caries: systematic review and network meta-analysis J Dent Res. 2019 Jan;98(1):14-26.

12. Tellez M, Gomez J, Kaur S, Pretty IA, Ellwood R, Ismail Al. Non-surgical management methods of noncavitated carious lesions. Community Dent Oral Epidemiol. 2013 Feb;41(1):79-96.

13. Elrashid AH, Alshaiji BS, Saleh SA, Zada KA, Baseer MA. Efficacy of resin infiltrate in noncavitated proximal carious lesions: a systematic review and metaanalysis. J Int Soc Prev Community Dent. 2019;9(3):211-8.

14. Chatzimarkou S, Koletsi D, Kavvadia K. The effect of resin infiltration on proximal caries lesions in primary and permanent teeth. A systematic review and metaanalysis of clinical trials. J Dent. 2018 Oct; 77:8-17.

15. Liang Y, Deng Z, Dai X, Tian J, Zhao W. Micro-invasive interventions for managing non-cavitated proximal caries of different depths: a systematic review and meta-analysis. Clin Oral Investig. 2018 Nov;22(8):2675-84.

16. Faghihian R, Shirani M, Tarrahi MJ, Zakizade M. Efficacy of the resin infiltration technique in preventing initial caries progression: a systematic review and metaanalysis. Pediatr Dent. 2019 Mar;41(2):88-94.

17. Meyer-Lueckel H, Bitter K, Paris S. Randomized controlled clinical trial on proximal caries infiltration: three-year follow-up. Caries Res. 2012;46(6):544-8.
18. Paris S, Hopfenmuller W, Meyer-Lueckel H. Resin infiltration of caries lesions: an efficacy randomized trial. J Dent Res. 2010;89(8):823-6.

19. Meyer-Lueckel H, Balbach A, Schikowsky C, Bitter K, Paris S. Pragmatic RCT on the efficacy of proximal caries infiltration. J Dent Res. 2016;95(5):531-6.

20. Arthur RA, Zenkner JE, Júnior JC d'Ornellas P, Correia RT, Alves LS, Maltz M. Proximal carious lesions infiltration-a 3-year follow-up study of a randomized controlled clinical trial. Clin Oral Investig. 2018;22(1):469-74.

21. Peters MC, Hopkins ARJ, Yu Q. Resin infiltration: An effective adjunct strategy for managing high caries risk-A within-person randomized controlled clinical trial. J Dent. 2018 Dec;79:24-30.

22. Agency for Toxic Substances and Disease Registry. Medical Management Guidelines for Hydrogen Chloride [Internet]. Toxic Substances Portal. 2014. Disponible en: https://wwwn.cdc.gov/TSP/MMG/MMGDetails.aspx?mmgid=758\&toxid=147

23. Roig-Vanaclocha A, Solá-Ruiz MF, Román-Rodríguez JL, Amengual-Lorenzo J, Pérez-Barquero JA, Agustín-Panadero R. Dental treatment of white spots and a description of the technique and digital quantification of the loss of enamel volume. Appl Sci. 2020;10(12):4369.

24. Schwendicke F, Meyer-Lueckel H, Stolpe M, Dörfer CE, Paris S. Costs and effectiveness of treatment alternatives for proximal caries lesions. PLoS One. 2014;9(1):e86992.

25. Schwendicke F, Paris S, Stolpe M. Detection and treatment of proximal caries lesions: milieu-specific cost--effectiveness analysis. J Dent. 2015;43(6):647-55.

26. Flink $H$, Tegelberg $A$, Arnetz JE, Birkhed D. Patient-reported negative experiences related to caries and its treatment among Swedish adult patients. BMC Oral Health. 2017;17(1):95.

27. Ismail Al, Pitts NB, Tellez M, et al. The International Caries Classification and Management System (ICCMSTM) an example of a caries management pathway. BMC Oral Health. 2015;15 Suppl 1(Suppl 1):S9.

28. Shirani M, Zakizade M. The efficacy of resin infiltration technique for inhibition of white spot lesion progression in primary and permanent teeth in comparison with other minimal invasive procedures. PROSPERO. 2017;CRD42017080432.

29. Puleio F, Lo Giudice R, Lo Giudice G. Is resin infiltration the best treatment in the aesthetic resolution of non-cavited white spot lesions? PROSPERO. 2020;CRD42020164187.

30. Reda S, Krois J. Sealing or infiltration of proximal carious lesions. PROSPERO. 2018;CRD42018080895

31. Najjar R, Halawani S, Sabbagh $H$, Nada M. The effect of fluoride varnish in treating proximal incipient carious lesions. PROSPERO. 2016;CRD42016030212.

32. Gawad F, Noha A, Nada W, Mervat R. Evaluation of esthetics and sensitivity after the use of resin infiltration in patients with white spot lesions: a systematic review. PROSPERO 2015;CRD42015027479.

33. Machado V, Botelho J, Manso C, Soveral M. Evaluation of the color stability, efficacy and effectiveness of infiltrative resin treatment measures for white lesions: a systematic review and meta-analysis. PROSPERO. 2019;CRD42019140860.

34. Vieira L. Non-invasive caries management: clinical study (NICM). Clin NCT04373356 [Internet]. 2020; Available from: https://clinicaltrials.gov/ct2/show/ NCT04373356 